\title{
Criteria for R-boundedness of operator families*
}

\author{
MARIA GIRARDI ${ }^{\dagger}$ \\ Department of Mathematics, University of South Carolina \\ Columbia, SC 29208, U.S.A. \\ e-mail: girardi@math.sc.edu \\ LUTZ WEIS \\ Mathematisches Institut I, Universität Karlsruhe \\ Englerstraße 2, 76128 Karlsruhe, Germany \\ e-mail: Lutz.Weis@math.uni-karlsruhe.de
}

\begin{abstract}
The main results state that large classes of convolution and Fourier multiplier operators are R-bounded. R-boundedness is of importance in connection with maximal regularity and functional calculus. Also shown is that smooth operator-valued functions have a R-bounded range, where the degree of smoothness depends on the geometry of the Banach space.
\end{abstract}

\section{INTRODUCTION}

Recently the notion of R-boundedness has played an important role in the functional analytic approach to partial differential equations. It is shown in [36] (see also $[2,3,7,8,14,15,23,26,35])$ that, for a sectorial operator $A$, R-boundedness of the set $\left\{\lambda(\lambda-A)^{-1}: \lambda \in \mathbb{C}_{+}\right\}$, i. e. R-sectoriality, characterizes maximal regularity. Rboundedness is also a very useful notion in connection with the $H^{\infty}$-calculus (see [23, $24]$ ). Furthermore it was shown in [36] (see also $[8,19,21,33]$ ) that R-boundedness provides a proper setting for boundedness theorems for operator-valued Fourier multipliers. Hence, workable criteria for R-boundedness are needed. It is already

* To appear: Recent Contributions to Evolution Equations, Lecture Notes in Math., Marcel Dekker.

$\dagger$ Girardi is supported in part by the Alexander von Humboldt Foundation.

$\ddagger$ Weis is supported in part by Landesforschungsschwerpunkt Evolutionsgleichungen des Landes Baden-Württenberg. 
known that sectorial operators with Gaussian estimates ( $[14,35])$, as well as large classes of partial differential operators ( $[7,15,25,26])$, are R-sectorial.

This leads to the natural question of whether each sectorial operator from a Banach space into itself is R-sectorial. Kalton and Lancien [22] answered this question in the negative; however, their counterexample is rather abstract and no reasonable sectorial differential operator which is not $\mathrm{R}$-sectorial is known. This note gives general criteria for R-boundedness, which may explain why the common operators of analysis are R-bounded and why it is so difficult to find counterexamples. This note's R-boundedness criteria are based on the observation that the very boundedness theorems for Fourier multipliers, which have R-boundedness in their assumption, improve themselves by a trick to give R-boundedness of classes of operators that satisfy these assumptions in a uniform way. This is possible provided $X$ has the UMD-property and Pisier's property $(\alpha)$. As a rule of thumb, the reflexive Banach spaces commonly appearing in analysis (such as subspaces of Sobolev spaces or Hardy spaces) have these properties. This idea is related to the fact (see [23]) that for a sectorial operator $A$ (on such spaces $X$ ) with an $H^{\infty}$ calculus, the set $\left\{f(A):\|f\|_{H^{\infty}} \leq 1\right\}$ is R-bounded. We think that our approach gives an unified way to prove R-boundedness for many families of operators relevant in applications and also can be applied to situations not considered here.

The main result of Section 3, Theorem 3.2, gives that the set of all Fourier multiplier operators that satisfy the Mihlin conditions in an uniform way form an R-bounded set. This is of particular interest for scalar-valued multiplier functions. Furthermore, Theorem 3.2 leads to (see Section 4) boundedness criteria for sets of convolution operators, which include the Gauß and Poisson semigroups. The results in Section 5 shows that the range of a sufficiently smooth function from $\mathbb{R}^{N}$ into $\mathcal{B}(X)$ is $\mathrm{R}$-bounded, where the Fourier type of $X$ is taken into account in order to reduce the required degree of smoothness. Then further applications to semigroups and resolvents are given. Section 2 collects needed definitions and facts.

Theorem 3.2 was presented by the authors at the TULKA seminar in February 2001. Independently and with different methods, A. Venni [34] has shown a special case of Theorem 3.2 .

\section{DEFINITIONS AND NOTATION}

Throughout this paper $X, Y$, and $Z$ are complex Banach spaces. $\mathcal{B}(X, Y)$ is the space of bounded linear operators from $X$ into $Y$; often $\mathcal{B}(X, X)$ is denoted by just $\mathcal{B}(X)$. The triple $(\Omega, \Sigma, \mu)$ is a $\sigma$-finite complete measure space; corresponding to it is the usual Bochner-Lebesgue space $L_{p}(\Omega ; X)$ of measurable functions from $\Omega$ into $X$ with finite $L_{p}(\Omega ; X)$-norm where $1 \leq p \leq \infty$; often $L_{p}(\Omega ; X)$ is denoted by just $L_{p}(X)$ or $L_{p}$ if confusion seems unlikely. If $\Omega=[0,1]$ or $\Omega=\mathbb{R}^{N}$ then $(\Omega, \Sigma, \mu)$ is understood to be the usual Lebesgue measure space. The Schwartz class $\mathcal{S}\left(\mathbb{R}^{N} ; X\right)$ is the space of rapidly decreasing smooth function from $\mathbb{R}^{N}$ to $X$. The sequence $\left\{r_{j}\right\}_{j=1}^{\infty}$ denotes a sequence of independent, symmetric, $\{1,-1\}-$ valued random variables on $[0,1]$, e.g. the Rademacher functions. $\mathbb{N}$ is the set of natural numbers while $\mathbb{N}_{0}=\mathbb{N} \cup\{0\}$.

$\mathrm{R}$-boundedness is the central notion of this paper.

DEFINITION 2.1. Let $\tau$ be a subset of $\mathcal{B}(X, Y)$ and $p \in[1, \infty)$. The num- 
ber $R_{p}(\tau)$ is the smallest of the constants $R \in[0, \infty]$ with the property that for each $n \in \mathbb{N}$ and subset $\left\{T_{j}\right\}_{j=1}^{n}$ of $\tau$ and subset $\left\{x_{j}\right\}_{j=1}^{n}$ of $X$,

$$
\left\|\sum_{j=1}^{n} r_{j}(\cdot) T_{j}\left(x_{j}\right)\right\|_{L_{p}([0,1] ; Y)} \leq R\left\|\sum_{j=1}^{n} r_{j}(\cdot) x_{j}\right\|_{L_{p}([0,1] ; X)} .
$$

The set $\tau$ is $R$-bounded provided $R_{p}(\tau)$ is finite for some (and thus then, by Kahane's inequality, for each) $p \in[1, \infty)$.

Thus a set $\tau$ is R-bounded provided Kahane's contraction principle holds for operator coefficients from $\tau$. Note that if $X$ and $Y$ are $q$-concave Banach lattices for some finite $q$ (e.g. $X=Y=L_{q}(\Omega ; \mathbb{C})$ where $\left.1 \leq q<\infty\right)$ then R-boundedness is equivalent to the square function estimate

$$
\left\|\left(\sum_{j=1}^{m}\left|T_{j} x_{j}\right|^{2}\right)^{1 / 2}\right\|_{Y} \leq R\left\|\left(\sum_{j=1}^{n}\left|x_{j}\right|^{2}\right)^{1 / 2}\right\|_{X}
$$

known from harmonic analysis (cf. [28, Thm. 1.d.6]). For basic properties of Rbounded sets and further references, see $[13,36]$.

The space $\operatorname{Rad}(X)$ provides a convenient way to view R-boundedness.

DEFINITION 2.2. For a Banach space $X$, define

$\operatorname{Rad}(X):=\left\{\widetilde{x}:=\left\{x_{j}\right\}_{j \in \mathbb{N}} \in X^{\mathbb{N}}: \sum_{j=1}^{n} r_{j}(\cdot) x_{j}\right.$ converges in $\left.L_{2}([0,1] ; X)\right\}$.

Often $\operatorname{Rad}(X)$ is denoted by just $\tilde{X}$.

When equipped with one of the following equivalent norms, where $1 \leq p<\infty$ :

$$
\left\|\left\{x_{j}\right\}_{j \in \mathbb{N}}\right\|_{\operatorname{Rad}_{p}(X)}:=\left\|\sum_{j \in \mathbb{N}} r_{j}(\cdot) x_{j}\right\|_{L_{p}([0,1] ; X)},
$$

$\operatorname{Rad}_{p}(X)$ is a Banach space. When confusion seems unlikely, $\operatorname{Rad}_{p}(X)$ is denoted by just $\operatorname{Rad}(X)$. Much can be found about $\operatorname{Rad}(X)$ in the literature (see, e.g. [16]).

FACT 2.3. A sequence $\left\{T_{j}\right\}_{j \in \mathbb{N}}$ from $\mathcal{B}(X, Y)$ is R-bounded if and only if the corresponding map

$$
\tilde{X} \ni\left\{x_{j}\right\}_{j \in \mathbb{N}} \stackrel{\widetilde{T}}{\longrightarrow}\left\{T_{j} x_{j}\right\}_{j \in \mathbb{N}} \in \tilde{Y}
$$

defines an element in $\mathcal{B}(\widetilde{X}, \widetilde{Y})$; in which case, $R_{p}\left(\left\{T_{j}\right\}_{j \in \mathbb{N}}\right)=\|\widetilde{T}\|_{\mathcal{B}\left(\operatorname{Rad}_{p}(X), \operatorname{Rad}_{p}(Y)\right)}$ for each $p \in[1, \infty)$.

Fubini's theorem yields the following useful fact.

FACT 2.4. Let $p \in[1, \infty)$. The mapping

$$
I_{X}: L_{p}\left(\Omega ; \operatorname{Rad}_{p}(X)\right) \rightarrow \operatorname{Rad}_{p}\left(L_{p}(\Omega ; X)\right)
$$

given by

$$
I_{X} f:=\left\{f_{j}\right\}_{j \in \mathbb{N}} \quad \text { for } \quad f(\cdot)=\left\{f_{j}(\cdot)\right\}_{j \in \mathbb{N}} \in L_{p}\left(\Omega ; \operatorname{Rad}_{p}(X)\right)
$$


is an isometry. Furthermore

$$
\widehat{f}=\left\{\widehat{f}_{j}\right\}_{j \in \mathbb{N}} \quad \text { and } \quad \check{f}=\left\{\check{f}_{j}\right\}_{j \in \mathbb{N}}
$$

for each $f(\cdot)=\left\{f_{j}(\cdot)\right\}_{j \in \mathbb{N}} \in L_{1}\left(\mathbb{R}^{N} ; \tilde{X}\right)$.

Considering R-boundedness in $\mathcal{B}(\widetilde{X}, \widetilde{Y})$ leads to estimating double random series; thus, an additional property of the Banach spaces $X$ and $Y$ is often needed.

DEFINITION 2.5. ( [32, Def. 2.1]) Let $\left\{\varepsilon_{k}\right\}_{k=1}^{\infty}$ (resp. $\left\{\varepsilon_{k}^{\prime}\right\}_{k=1}^{\infty}$ ) be a sequence of independent, symmetric, $\{1,-1\}$-valued random variables on some probability space $(\Omega, \Sigma, \mu)\left(\operatorname{resp} .\left(\Omega^{\prime}, \Sigma^{\prime}, \mu^{\prime}\right)\right)$ with $\left\{\varepsilon_{k}\right\}_{k=1}^{\infty}$ and $\left\{\varepsilon_{k}^{\prime}\right\}_{k=1}^{\infty}$ independent. For a Banach space $X$ and $1 \leq p<\infty$, the number $\alpha_{p}(X)$ is the smallest of the constants $\alpha \in[0, \infty]$ with the property that

$$
\left\|\sum_{j, k=1}^{n} \alpha_{j k} \varepsilon_{j} \varepsilon_{k}^{\prime} x_{j k}\right\|_{L_{p}\left(\Omega \times \Omega^{\prime} ; X\right)} \leq \alpha\left\|\sum_{j, k=1}^{n} \varepsilon_{j} \varepsilon_{k}^{\prime} x_{j k}\right\|_{L_{p}\left(\Omega \times \Omega^{\prime} ; X\right)}
$$

for each $n \in \mathbb{N}$ and subset $\left\{x_{j k}\right\}_{j, k=1}^{n}$ of $X$ and choices $\left\{\alpha_{j k}\right\}_{j, k=1}^{n}$ of signs $\{1,-1\}$. The space $X$ has property $(\alpha)$ provided $\alpha_{p}(X)$ is finite for some (and thus then, by Kahane's inequality, for each) $p \in[1, \infty)$.

Each subspace [32] of a Banach function space of finite cotype (e.g. a subspace of $L_{p}(\Omega ; \mathbb{C})$ with $\left.1 \leq p<\infty\right)$ has property $(\alpha)$. If $X$ has property $(\alpha)$, then so does $L_{q}(\Omega ; X)$ for $1 \leq q<\infty$. The estimate in (2.3) extends to R-bounded sets.

FACT 2.6. (CF. [13, Lemma 2.3.3]) Let $X$ and $Y$ be Banach spaces enjoying property $(\alpha)$ and $\tau$ be an $R$-bounded subset of $\mathcal{B}(X, Y)$. Then for $p \in[1, \infty)$

$$
\left\|\sum_{j, k=1}^{n} \varepsilon_{j} \varepsilon_{k}^{\prime} T_{j k} x_{j k}\right\|_{L_{p}\left(\Omega \times \Omega^{\prime} ; Y\right)} \leq \alpha_{p}(X) \alpha_{p}(Y) R_{p}(\tau)\left\|\sum_{j, k=1}^{n} \varepsilon_{j} \varepsilon_{k}^{\prime} x_{j k}\right\|_{L_{p}\left(\Omega \times \Omega^{\prime} ; X\right)}
$$

for each $n \in \mathbb{N}$ and subset $\left\{T_{j k}\right\}_{j, k=1}^{n}$ of $\tau$ and subset $\left\{x_{j k}\right\}_{j, k=1}^{n}$ of $X$.

The UMD property is often needed for vector-valued multiplier theorems.

DEFINITION 2.7. A Banach space $X$ is a $U M D$ space provided the Hilbert transform

$$
H f(t)=P V-\int \frac{f(s)}{t-s} d s \quad \text { for } \quad f \in \mathcal{S}(X)
$$

extends to a bounded linear operator on $L_{p}(\mathbb{R} ; X)$ for some (and thus then for each) $p \in(1, \infty)$.

Thus $X$ is a UMD space if and only if $m: \mathbb{R} \backslash\{0\} \rightarrow \mathcal{B}(X)$ given by

$$
m(t)=\operatorname{sign}(t) I_{X}
$$

is a Fourier multiplier on $L_{p}(\mathbb{R} ; X)$ for some (and thus then for each) $p \in(1, \infty)$. Closed subspaces of, the dual of, and quotients spaces of a UMD space are UMD spaces. Each Hilbert space is a UMD space. If $X$ is a UMD space and $q \in(1, \infty)$, then $L_{q}(\Omega ; X)$ is also a UMD space. 
For $f \in L_{1}\left(\mathbb{R}^{N} ; X\right)$ we denote by $\widehat{f}$ or $\mathcal{F} f$ the Fourier transform of $f$, i.e.,

$$
\widehat{f}(t) \equiv \mathcal{F}(f)(t):=\int_{\mathbb{R}^{N}} e^{-i t \cdot s} f(s) d s .
$$

In the vector-valued setting the Hausdorff-Young inequality does not hold in general. Thus one considers the following class of Banach spaces.

DEFINITION 2.8. ( [30]) A Banach space $X$ has Fourier type $p$, where $p \in[1,2]$, if the Fourier transform $\mathcal{F}$ defines a bounded linear operator from $L_{p}\left(\mathbb{R}^{N} ; X\right)$ to $L_{p^{\prime}}\left(\mathbb{R}^{N} ; X\right)$ for some (and thus then for each) $N \in \mathbb{N}$. The Fourier type constant $\mathcal{F}_{p, N}(X)$ of $X$ is then the norm of $\mathcal{F} \in \mathcal{B}\left(L_{p}\left(\mathbb{R}^{N} ; X\right), L_{p^{\prime}}\left(\mathbb{R}^{N} ; X\right)\right)$.

The simple estimate $\|\mathcal{F} f(t)\|_{X} \leq\|f\|_{L_{1}(X)}$ shows that each Banach space $X$ has Fourier type 1 with $\mathcal{F}_{1, N}(X)=1$. The notion becomes more restrictive as $p$ increases to 2. A Banach space has Fourier type 2 if and only if $X$ is isomorphic to a Hilbert space [27]. A space $L_{q}(\Omega ; \mathbb{R})$ has Fourier type $p=\min \left(q, q^{\prime}\right)$ [30]. If $X$ have Fourier type $p \in[1,2]$ and $p \leq q \leq p^{\prime}$, then $\mathcal{F}_{p, N}(X)=\mathcal{F}_{p, N}\left(X^{*}\right)=$ $\mathcal{F}_{p, N}\left(L_{q}\left(\mathbb{R}^{N} ; X\right)\right)$ for each $N \in \mathbb{N}$ (cf. [18]). Each closed subspace (by definition) and quotient space (by duality) of a Banach space $X$ has the same Fourier type as $X$.

REMARK 2.9. Recall the following relationships between property $(\alpha)$, UMD spaces, Fourier type, and $\operatorname{Rad}(X)$.

a) Property $(\alpha)$ and the UMD property are independent. An $L_{1}(\Omega ; \mathbb{C})$ space has property $(\alpha)$. However, infinite dimensional $L_{1}(\Omega ; \mathbb{C}$ ) spaces (as well as $C(K)$ spaces) are not UMD spaces. The Schatten classes $\mathcal{S}_{p}$ have UMD for $p \in(1, \infty)$. However, an infinite dimensional $\mathcal{S}_{p}$ does not have property $(\alpha)$ when $1 \leq p \leq \infty$ and $p \neq 2$.

b) A UMD space has some non-trivial Fourier type $p>1$. Indeed, a UMD space has a uniformly convex renorming [10]. A space with a uniformly convex renorming is reflexive and B-convex. A B-convex Banach space has some non-trivial Fourier type $p>1[9,12]$.

c) If $X$ has property $(\alpha)$ (resp. the UMD property, Fourier type $p$ ), then so does $\operatorname{Rad}(X)$. This follows from viewing $\operatorname{Rad}(X)$ as a subspace of $L_{2}([0,1] ; X)$.

\section{FOURIER MULTIPLIER OPERATORS}

Let $q \in(1, \infty)$. A bounded measurable function $M: \mathbb{R}^{N} \backslash\{0\} \rightarrow \mathcal{B}(X, Y)$ is a Fourier multiplier on $L_{q}\left(\mathbb{R}^{N} ; X\right)$ provided the corresponding Fourier multiplier operator $T_{M}$, defined by

$$
T_{M} f:=[M(\cdot) \widehat{f}(\cdot)]^{\vee} \quad \text { for } \quad f \in \mathcal{S}\left(\mathbb{R}^{N} ; X\right),
$$

extends to a bounded operator $T_{M} \in \mathcal{B}\left(L_{q}\left(\mathbb{R}^{N} ; X\right), L_{q}\left(\mathbb{R}^{N} ; Y\right)\right)$.

FACT 3.1. Let $\tau$ be a R-bounded subset of $\mathcal{B}(X, Y)$ and $q \in(1, \infty)$. It is known that $M$ is a Fourier multiplier on $L_{q}\left(\mathbb{R}^{N} ; X\right)$ if $X$ and $Y$ are UMD spaces with 
Fourier type $p \in[1,2]$ and $M$ belongs to one of the following symbol classes:

$$
\begin{aligned}
& \mathcal{M}^{l}(\tau):= \\
& \quad\left\{M: \mathbb{R}^{N} \backslash\{0\} \rightarrow \mathcal{B}(X, Y):|t|^{|\alpha|} M^{(\alpha)}(t) \in \tau \text { for } t \neq 0, \alpha \in \mathbb{N}_{0}^{N},|\alpha| \leq l\right\}
\end{aligned}
$$

for some $l \in \mathbb{N}$ where $l>N / p($ see $[19,21,33,36])$;

$$
\begin{aligned}
& \mathcal{H}^{N}(\tau):= \\
& \quad\left\{M: \mathbb{R}^{N} \backslash\{0\} \rightarrow \mathcal{B}(X, Y): t^{\alpha} M^{(\alpha)}(t) \in \tau \text { for } t \neq 0, \alpha \in \mathbb{N}_{0}^{N}, \alpha \leq(1,1, \ldots, 1)\right\}
\end{aligned}
$$

provided $X$ and $Y$ have property $(\alpha)$ (see [33]);

$$
\begin{aligned}
& \mathcal{N}^{r}(\tau):= \\
& \left\{M: \mathbb{R} \backslash\{0\} \rightarrow \mathcal{B}(X, Y): M(t) \in \tau,|t|^{r} \frac{M(t+s)-M(t)}{|s|^{r}} \in \tau \text { for } s, t \neq 0\right\}
\end{aligned}
$$

for some $r \in(1 / p, 1)$ (see [19]). If $X=Y=\mathbb{C}$ (i.e., scalar-valued multiplier functions) and $\tau$ is the unit ball of $\mathbb{C}$, then the above classes are denoted by just $\mathcal{M}^{l}, \mathcal{H}^{N}$, and $\mathcal{N}^{r}$.

Fact 2.6 leads to our main criteria for R-boundedness of sets of Fourier multiplier operators.

THEOREM 3.2. Let $X$ and $Y$ be UMD Banach spaces having property $(\alpha)$ and Fourier type $p$. Let $l=[N / p]+1$ and $r \in(1 / p, 1)$. Let $\tau$ be a R-bounded subset of $\mathcal{B}(X, Y)$. Then the sets of Fourier multiplier operators

$$
\begin{aligned}
\mathcal{M} & :=\left\{T_{M} \in \mathcal{B}\left(L_{q}\left(\mathbb{R}^{N} ; X\right), L_{q}\left(\mathbb{R}^{N} ; Y\right)\right): M \in \mathcal{M}^{l}(\tau)\right\} \\
\mathcal{H} & :=\left\{T_{M} \in \mathcal{B}\left(L_{q}\left(\mathbb{R}^{N} ; X\right), L_{q}\left(\mathbb{R}^{N} ; Y\right)\right): M \in \mathcal{H}^{N}(\tau)\right\} \\
\mathcal{N} & :=\left\{T_{M} \in \mathcal{B}\left(L_{q}\left(\mathbb{R}^{1} ; X\right), L_{q}\left(\mathbb{R}^{1} ; Y\right)\right): M \in \mathcal{N}^{r}(\tau)\right\}
\end{aligned}
$$

are R-bounded for each $q \in(1, \infty)$.

PROOF. Fix $q \in(1, \infty)$ To simplify notation, denote $L_{q}\left(\mathbb{R}^{N} ; Z\right)$ by just $L_{q}(Z)$ and $\operatorname{Rad}_{q}(Z)$ by just $\widetilde{Z}$ for the assorted Banach spaces $Z$ appearing.

Let $\left\{M_{j}\right\}_{j=1}^{n}$ be a subset of $\mathcal{M}^{l}(\tau)$ (resp. $\left.\mathcal{H}^{N}(\tau), \mathcal{N}^{r}(\tau)\right)$ and set $M_{j}:=0$ for $j>n$. Define

$$
\widetilde{T} \in \mathcal{B}\left(\widetilde{L_{q}(X)}, \widetilde{L_{q}(Y)}\right)
$$

by

$$
\widetilde{T} \widetilde{g}=\left\{T_{M_{j}} g_{j}\right\}_{j=1}^{\infty} \quad \text { for } \quad \widetilde{g}=\left\{g_{j}\right\}_{j=1}^{\infty} \in \widetilde{L_{q}(X)} .
$$

Note that $\|\widetilde{T}\|=R_{q}\left(\left\{T_{M_{j}}\right\}_{j=1}^{n}\right)<\infty$. By Fact 2.3 , it suffices to show that there exists a constant $C_{*}$ that depends only on $R_{q}(\tau), X, Y, q, p$, and $N$ (but not on the particular chosen $\left.\left\{M_{j}\right\}_{j=1}^{n}\right)$ such that

$$
\|\widetilde{T}\|_{\mathcal{B}\left(\widetilde{L_{q}(X)}, \widetilde{L_{q}(Y)}\right)} \leq C_{*} .
$$


For then $R_{q}(\mathcal{M})$ (resp. $\left.R_{q}(\mathcal{H}), R_{q}(\mathcal{N})\right)$ is bounded above by $C_{*}$.

Towards this, define

$$
\widetilde{M}: \mathbb{R}^{N} \backslash\{0\} \rightarrow \mathcal{B}(\widetilde{X}, \widetilde{Y})
$$

by

$$
[\widetilde{M}(\cdot)]\left(\left\{x_{j}\right\}_{j \in \mathbb{N}}\right):=\left\{M_{j}(\cdot) x_{j}\right\}_{j \in \mathbb{N}} \quad \text { for } \quad\left\{x_{j}\right\}_{j \in \mathbb{N}} \in \widetilde{X} .
$$

Note that $\|\widetilde{M}(t)\|=R_{q}\left(\left\{M_{j}(t)\right\}_{j=1}^{n}\right) \leq R_{q}(\tau)$ for each $t \in \mathbb{R}^{N} \backslash\{0\}$. To show (3.1), it suffices to show that $\widetilde{M}$ is a Fourier multiplier on $L_{q}(\widetilde{X})$ with

$$
\left\|T_{\widetilde{M}}\right\|_{\mathcal{B}\left(L_{q}(\widetilde{X}), L_{q}(\widetilde{Y})\right)} \leq C_{*} .
$$

Indeed, for then the diagram

$$
\begin{array}{cc}
\operatorname{Rad}_{q}\left(L_{q}(X)\right) & \stackrel{\widetilde{T}}{\longrightarrow} \\
I_{X} \uparrow & \operatorname{Rad}_{q}\left(L_{q}(Y)\right) \\
\left.L_{q} \operatorname{Rad}_{q}(X)\right) \stackrel{\operatorname{Ra}_{\widetilde{M}}}{\longrightarrow} & L_{q}\left(\operatorname{Rad}_{q}(Y)\right)
\end{array}
$$

commutes since if $f(\cdot)=\left\{f_{j}(\cdot)\right\}_{j \in \mathbb{N}} \in \mathcal{S}\left(\mathbb{R}^{N} ; \widetilde{X}\right)$ then $T_{\widetilde{M}} f=[\widetilde{M}(\cdot) \widehat{f}(\cdot)]^{\vee}$ and so, with the help of $(2.2)$,

$$
I_{Y} T_{\widetilde{M}} f=\left\{T_{M_{j}} f_{j}\right\}_{j \in \mathbb{N}}=\widetilde{T} I_{X} f ; \text { hence, } \quad\left\|T_{\widetilde{M}}\right\|=\|\widetilde{T}\| .
$$

First consider the set $\mathcal{M}$. Note that the set

$$
\left\{|t|^{|\alpha|}\left(D^{\alpha} \widetilde{M}\right)(t) \in \mathcal{B}(\tilde{X}, \tilde{Y}): t \in \mathbb{R}^{N} \backslash\{0\}, \alpha \in \mathbb{N}_{0}^{N},|\alpha| \leq l\right\}
$$

is R-bounded; indeed, for any fixed subsets

$$
\left\{t_{j}\right\}_{j=1}^{m} \subset \mathbb{R}^{N} \backslash\{0\} \quad \text { and } \quad\left\{\widetilde{x}_{j}:=\left\{x_{k}^{j}\right\}_{k \in \mathbb{N}}\right\}_{j=1}^{m} \subset \widetilde{X}
$$

and $\alpha \in \mathbb{N}_{0}$ with $|\alpha| \leq l$, note that $D^{\alpha} \widetilde{M}=\left\{D^{\alpha} M_{k}\right\}_{k \in \mathbb{N}}$ and so for

$$
N_{k}(t):=|t|^{|\alpha|} D^{\alpha} M_{k}(t) \quad \text { and } \quad \tilde{N}(t):=\left\{N_{k}(t)\right\}_{k \in \mathbb{N}},
$$


by Fact 2.6,

$$
\begin{aligned}
\left\|\sum_{j=1}^{m} r_{j}(\cdot) \widetilde{N}\left(t_{j}\right) \widetilde{x}_{j}\right\|_{L_{q}([0,1] ; \widetilde{Y})}^{q} & \int_{[0,1][0,1]^{\prime}} \int_{k=1}\left\|\sum_{j=1}^{n} \sum_{k}^{m} r_{k}^{\prime}\left(u^{\prime}\right) r_{j}(u) N_{k}\left(t_{j}\right) x_{k}^{j}\right\|_{Y}^{q} d u^{\prime} d u \\
& \leq C_{0}^{q} \int_{[0,1][0,1]^{\prime}}\left\|\sum_{k=1}^{n} \sum_{j=1}^{m} r_{k}^{\prime}\left(u^{\prime}\right) r_{j}(u) x_{k}^{j}\right\|_{X}^{q} d u^{\prime} d u \\
& \leq C_{0}^{q}\left\|\sum_{j=1}^{m} r_{j}(\cdot) \widetilde{x}_{j}\right\|_{L_{q}([0,1] ; \widetilde{X})}^{q},
\end{aligned}
$$

where $C_{0}=\alpha_{q}(X) \alpha_{q}(Y) R_{q}(\tau)$. Thus by Remark 2.9c) and Fact 3.1, $\widetilde{M}$ is a Fourier multiplier on $L_{q}(\widetilde{X})$ with $T_{\widetilde{M}}$ bounded by some constant $C_{*}$ that depends only on the items claimed.

The proofs for $\mathcal{H}$ and $\mathcal{N}$ are similar. The only difference is that for the estimate in (3.2) one considers $\alpha \in \mathbb{N}_{0}$ with $\alpha \leq(1,1, \ldots, 1)$ and uses $N_{k}(t):=$ $t^{\alpha}\left(D^{\alpha} M_{k}\right)(t)$ in the case of $\mathcal{H}$ while one considers $\left\{s_{j}\right\}_{j=1}^{m} \subset \mathbb{R}^{N} \backslash\{0\}$ also and uses $N_{k}(s, t):=|t / s|^{r}\left[M_{k}(t+s)-M_{k}(t)\right]$ or $N_{k}(t)=M_{k}(t)$ in the case of $\mathcal{N}$.

Since $X=Y=\mathbb{C}$ have $U M D$, property $(\alpha)$, and Fourier type 2, an interesting R-boundedness result follows for scalar-valued Fourier multipliers on $L_{q}\left(\mathbb{R}^{N} ; \mathbb{C}\right)$.

COROLLARY 3.3. Let $l=[N / 2]+1$ and choose $r \in(1 / 2,1)$. Then the sets of Fourier multiplier operators $\left\{T_{M}: M \in \mathcal{M}^{l}\right\},\left\{T_{M}: M \in \mathcal{H}^{N}\right\}$, and $\left\{T_{M}: M \in \mathcal{N}^{r}\right\}$ are R-bounded on $L_{q}\left(\mathbb{R}^{N} ; \mathbb{C}\right.$ ) for each $1<q<\infty$ (of course, $N=1$ in the latter case).

\section{CONVOLUTION OPERATORS}

The theorem in the previous section gives that certain families of Fourier multiplier operators are R-bounded. The results of this section give that certain families of convolution operators are R-bounded. Recall that for a kernel function $k \in L_{1}\left(\mathbb{R}^{N} ; \mathcal{B}(X, Y)\right)$ and $q \in[1, \infty]$, one can define the corresponding convolution operator $K_{k} \in \mathcal{B}\left(L_{q}\left(\mathbb{R}^{N} ; X\right), L_{q}\left(\mathbb{R}^{N} ; Y\right)\right)$ by

$$
K_{k} f(t):=\int_{\mathbb{R}^{N}} k(t-s) f(s) d s \quad \text { for a.e. } \quad t \in \mathbb{R}^{N} .
$$

Note that it follows from Lemma 4.1 that $\left\{\widehat{k}(t): t \in \mathbb{R}^{N}\right\}$ is R-bounded in $\mathcal{B}(X, Y)$.

LEMMA 4.1. Let $X$ and $Y$ be arbitrary Banach spaces.

a) Let $N: \Omega \rightarrow \mathcal{B}(X, Y)$ be strongly integrable on the measure space $(\Omega, \Sigma, \mu)$. 
Thus there is a constant $A$ so that

$$
\int_{\Omega}\|N(\omega) x\|_{Y} d \mu(\omega) \leq A\|x\|_{X} \quad \text { for each } \quad x \in X
$$

Then

$$
\tau:=\left\{X \ni x \rightarrow \int_{\Omega} h(\omega) N(\omega) x d \mu(\omega) \in Y: h \in L_{\infty}(\Omega ; \mathbb{C}),\|h\|_{\infty} \leq 1\right\}
$$

is an R-bounded subset of $\mathcal{B}(X, Y)$ with $R_{1}(\tau) \leq 2 A$.

b) If $N \in L_{1}(\Omega ; \mathcal{B}(X, Y))$, then

$$
\begin{array}{r}
R_{1}\left(\left\{\int_{\Omega} h(\omega) N(\omega) d \mu(\omega) \in \mathcal{B}(X, Y): h \in L_{\infty}(\Omega ; \mathbb{C}),\|h\|_{\infty} \leq 1\right\}\right) \leq \\
2\|N\|_{L_{1}(\Omega ; \mathcal{B}(X, Y))} .
\end{array}
$$

PROOF. Clearly it suffices to show just part a). For $h \in L_{\infty}(\Omega ; \mathbb{C})$, let

$$
T_{h} x=\int_{\Omega} h(\omega) N(\omega) x d \mu(\omega)
$$

so that $T_{h} \in \mathcal{B}(X, Y)$ with $\left\|T_{h}\right\|_{\mathcal{B}(X, Y)} \leq A\|h\|_{\infty}$. For $h_{1}, \ldots, h_{n} \in L_{\infty}(\Omega ; \mathbb{C})$ with $\left\|h_{i}\right\|_{\infty} \leq 1$ and $x_{1}, \ldots, x_{n} \in X$

$$
\begin{aligned}
\int_{[0,1]}\left\|\sum_{j=1}^{n} r_{j}(t) T_{h_{j}}\left(x_{j}\right)\right\|_{Y} d t & \int_{[0,1]}\left\|\int_{\Omega}\left(\sum_{j=1}^{n} r_{j}(t) h_{j}(\omega) N(\omega) x_{j}\right) d \mu(\omega)\right\|_{Y} d t \\
& \leq \int_{\Omega} \int\left\|\sum_{j=1}^{n} r_{j}(t) h_{j}(\omega) N(\omega) x_{j}\right\|_{Y} d t d \mu(\omega) \\
& \leq 2 \iint_{\Omega}\left\|\sum_{j=1}^{n} r_{j}(t) N(\omega) x_{j}\right\|_{Y} d t d \mu(\omega) \\
& \left.=2 \iint_{[0,1]}\left\|N_{\Omega(\omega)}\left[\sum_{j=1}^{n} r_{j}(t) x_{j}\right]\right\|_{Y} d \mu(\omega)\right) d t \\
& \leq 2 \int_{[0,1]}\left\|\sum_{j=1}^{n} r_{j}(t) x_{j}\right\|_{X} d t
\end{aligned}
$$

by Kahane's contraction principle and (4.2).

Lemma 4.1 leads to an R-boundedness criterion for certain families of kernels. 
PROPOSITION 4.2. Let $X$ and $Y$ be UMD spaces with property $(\alpha)$. Let the kernel function $k: \mathbb{R}^{N} \rightarrow \mathcal{B}(X, Y)$ satisfy, for each $\alpha \in \mathbb{N}_{0}^{\mathbb{N}}$ with $\alpha \leq(1,1, \ldots, 1)$,

$$
\int_{\mathbb{R}^{N}}\left|t^{\alpha}\right|\left\|D^{\alpha} k(t)\right\|_{\mathcal{B}(X, Y)} d t<\infty
$$

Then the family $\left\{K_{\gamma} \in \mathcal{B}\left(L_{q}\left(\mathbb{R}^{N} ; X\right), L_{q}\left(\mathbb{R}^{N} ; Y\right)\right): \gamma \in \mathbb{R}\right\}$, defined by

$$
\left(K_{\gamma} f\right)(t):=\int \gamma^{N} k(\gamma(t-s)) f(s) d s \quad \text { for } \quad f \in \mathcal{S}\left(\mathbb{R}^{N} ; X\right),
$$

is R-bounded for each $q \in(1, \infty)$.

REMARK 4.3. For $X=Y=\mathbb{C}$, Proposition 4.2 reduces to: if $k \in \mathbb{R}^{N} \rightarrow \mathbb{C}$ satisfies

$$
\int_{\mathbb{R}^{N}}\left|t^{\alpha} D^{\alpha} k(t)\right| d t<\infty \quad \text { for each } \quad \alpha \in \mathbb{N}_{0}^{\mathbb{N}} \quad \text { with } \quad \alpha \leq(1,1, \ldots, 1)
$$

then the family $\left\{K_{\gamma} \in \mathcal{B}\left(L_{q}\left(\mathbb{R}^{N} ; \mathbb{C}\right)\right): \gamma \in \mathbb{R}\right\}$ is R-bounded for each $q \in(1, \infty)$. REMARK 4.4. The following variant of Proposition 4.2 is also true. First replace (4.3) by

$$
\int_{\mathbb{R}^{N}}\left|t^{\alpha}\right|\left\|D^{\alpha} k(t) x\right\|_{Y} d t \leq A\|x\|_{X} \quad \text { for each } x \in X .
$$

Note that then $k$ is strongly integrable and so the function $M: \mathbb{R}^{N} \rightarrow \mathcal{B}(X, Y)$, given by

$$
M(t) x:=[k(\cdot) x]^{\wedge}(t) \quad \text { for } t \in \mathbb{R}^{N} \text { and } x \in X,
$$

is well-defined. Assume further that the distributional derivatives $D^{\alpha} M$ are represented by functions for each $\alpha \in \mathbb{N}_{0}^{\mathbb{N}}$ with $\alpha \leq(1,1, \ldots, 1)$. Then the conclusion of Proposition 4.2 holds.

Proof of Proposition 4.2 and Remark 4.4. Let $X$ and $Y$ be UMD spaces with property $(\alpha)$. Let $k$ satisfy either $(4.3)$ or $\left(4.3^{\prime}\right)$ and $M$ be as in (4.4). Define $k_{x}, M_{x}: \mathbb{R}^{N} \rightarrow Y$ by

$$
k_{x}(\cdot):=k(\cdot) x \quad M_{x}(\cdot):=M(\cdot) x=\widehat{k}_{x}(\cdot)
$$

for each $x \in X$.

Fix $\alpha \in \mathbb{N}_{0}^{\mathbb{N}}$ with $\alpha \leq(1,1, \ldots, 1)$. Then for each $x \in X$

$$
D^{\alpha}\left(s^{\alpha} k_{x}(s)\right)=\sum_{\gamma \leq \alpha}\left(\begin{array}{l}
\alpha \\
\gamma
\end{array}\right) D^{\alpha-\gamma}\left(s^{\alpha}\right) D^{\gamma}\left(k_{x}(s)\right)=\sum_{\gamma \leq \alpha} C_{\alpha, \gamma} s^{\gamma} D^{\gamma}\left(k_{x}(s)\right)
$$

and

$$
(-1)^{|\alpha|}\left[D^{\alpha}\left(s^{\alpha} k_{x}(s)\right)\right]^{\curlywedge}(t)=(-i)^{|\alpha|} t^{\alpha}\left[s^{\alpha} k_{x}(s)\right]^{\curlywedge}(t)=t^{\alpha} D^{\alpha} M_{x}(t) .
$$


Thus if $k$ satisfies (4.3), then $D^{\alpha}\left(s^{\alpha} k(s)\right) \in L_{1}(\mathcal{B}(X, Y))$ and so the distributional derivative $D^{\alpha} M$ is represented as a function. Thus it suffices to show just Remark 4.4; so, assume the setting of Remark 4.4.

By (4.6) and (4.5)

$$
t^{\alpha} D^{\alpha} M_{x}(t)=(-1)^{|\alpha|} \sum_{\gamma \leq \alpha} C_{\alpha, \gamma} \int_{\mathbb{R}^{N}} h_{t}(s)\left[s^{\gamma} D^{\gamma} k_{x}(s)\right] d s
$$

where $h_{t}(s):=e^{-i t \cdot s}$. Thus by $\left(4.3^{\prime}\right)$ and Lemma 4.1 , the set

$$
\tau_{\alpha}:=\left\{t^{\alpha} D^{\alpha} M(t): t \in \mathbb{R}^{N}\right\}
$$

is R-bounded.

Fix $\gamma \in \mathbb{R}^{N} \backslash\{0\}$. Then $M_{\gamma}: \mathbb{R}^{N} \rightarrow \mathcal{B}(X, Y)$, defined below, satisfies

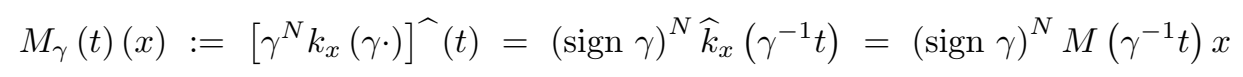

and so

$$
t^{\alpha} D^{\alpha} M_{\gamma}(t)=(\operatorname{sign} \gamma)^{N}\left(\gamma^{-1} t\right)^{\alpha} D^{\alpha} M\left(\gamma^{-1} t\right) \in \pm \tau_{\alpha}
$$

for each $t \in \mathbb{R}^{N}$. Hence $M_{\gamma} \in \mathcal{H}^{N}\left(\cup_{\alpha \leq(1, \ldots, 1)} \pm \tau_{\alpha}\right)$ and so $M_{\gamma}$ is a Fourier multiplier on $L_{q}\left(\mathbb{R}^{N} ; X\right)$ by Fact 3.1. Furthermore, Theorem 3.2 gives that $\left\{K_{\gamma}: \gamma \in \mathbb{R}\right\}$ is R-bounded.

R-boundedness of the Gauß and Poisson semigroups follows from Proposition 4.2.

EXAMPLE 4.5. Keeping with Proposition 4.2's notation, fix $\gamma>0$. If

$$
k(s):=(4 \pi)^{-1 / 2} \exp \left(\frac{-|s|^{2}}{4}\right),
$$

then

$$
\begin{gathered}
\left(K_{\gamma^{-1 / 2}} f\right)(t)=(4 \pi \gamma)^{-N / 2} \int_{\mathbb{R}^{N}} \exp \left(\frac{-|s|^{2}}{4 \gamma}\right) f(t-s) d s=\left(e^{\gamma \Delta} f\right)(t) . \\
\text { If } k(s):=C_{N}\left(1+|s|^{2}\right)^{-(N+1) / 2} \text { where } C_{N}:=\Gamma\left(\frac{N+1}{2}\right) \pi^{-(N+1) / 2} \text { then } \\
\left(K_{\gamma^{-1}} f\right)(t)=C_{N} \int_{\mathbb{R}^{N}} \frac{\gamma}{\left(\gamma^{2}+|s|^{2}\right)^{(N+1) / 2}} f(t-s) d s=\left(e^{-\gamma \sqrt{-\Delta}} f\right)(t) .
\end{gathered}
$$

Since both functions $k$ satisfy (4.3), Remark 4.3 gives that the Gauß semigroup $\left\{e^{\gamma \Delta}: \gamma>0\right\}$ and the Poisson semigroup $\left\{e^{-\gamma \sqrt{-\Delta}}: \gamma>0\right\}$ are R-bounded in $\mathcal{B}\left(L_{q}\left(\mathbb{R}^{N} ; \mathbb{C}\right)\right)$ for each $q \in(1, \infty)$.

A more general criterion for R-boundedness of a family of convolution operators is now formulated. For this, let $\tau$ be a R-bounded subset of $\mathcal{B}(X, Y)$ and $\tau_{0}$ be the closure (in the strong operator topology) of the complex absolute convex hull of $\tau$. Note that $\tau_{0}$ is also R-bounded (indeed, $R_{p}\left(\tau_{0}\right) \leq 2 R_{p}(\tau)$ ). Let $\|\cdot\|_{\tau}: \mathcal{B}(X, Y) \rightarrow[0, \infty]$ be the Minkowski functional of $\tau_{0}$, i.e.,

$$
\|S\|_{\tau}:=\inf \left\{\lambda \geq 0: S \in \lambda \tau_{0}\right\} .
$$


Define

$\mathcal{K}^{N}(\tau):=\left\{k: \mathbb{R}^{N} \rightarrow \mathcal{B}(X, Y): \int_{\mathbb{R}^{N}}\left\|t^{\alpha} D^{\alpha} k(t)\right\|_{\tau} d t \leq 1\right.$ for each $\left.\alpha \leq(1, \ldots, 1)\right\}$.

Note that if $k \in \mathcal{K}^{n}(\tau)$, then $k$ satisfies (4.3); in particular, the convolution operator $K_{k}$, as defined in $(4.1)$, is in $\mathcal{B}\left(L_{q}\left(\mathbb{R}^{N} ; X\right), L_{q}\left(\mathbb{R}^{N} ; Y\right)\right)$ for each $q \in(1, \infty)$.

THEOREM 4.6. Let $X$ and $Y$ be UMD spaces with property $(\alpha)$ and $\tau$ be an R-bounded subset of $\mathcal{B}(X, Y)$. Then the family of convolution operators

$$
\left\{K_{k} \in \mathcal{B}\left(L_{q}\left(\mathbb{R}^{N} ; X\right), L_{q}\left(\mathbb{R}^{N} ; Y\right)\right): k \in \mathcal{K}^{N}(\tau)\right\}
$$

is R-bounded for each $q \in(1, \infty)$.

REMARK 4.7. Let $X=Y=\mathbb{C}$ (i.e. scalar-valued kernels) and $\tau$ be the unit ball of $\mathbb{C}$ (so $\left.\|\cdot\|_{\tau}=|\cdot|\right)$. Then Theorem 4.6 implies that

$$
\left\{K_{k} \in \mathcal{B}\left(L_{q}\left(\mathbb{R}^{N} ; \mathbb{C}\right)\right): \int_{\mathbb{R}^{N}}\left|t^{\alpha} D^{\alpha} k(t)\right| d t \leq 1 \quad \text { for each } \alpha \leq(1, \ldots, 1)\right\}
$$

is R-bounded for each $q \in(1, \infty)$.

Proof of Theorem 4.6 Fix $k \in \mathcal{K}^{N}(\tau)$. Then, keeping with the notation of Proposition 4.2,

$$
t^{\alpha} D^{\alpha} M(t)=(-1)^{|\alpha|} \sum_{\gamma \leq \alpha} C_{\alpha, \gamma} \int_{\mathbb{R}^{N}} e^{-i t \cdot s}\left[s^{\gamma} D^{\gamma} k(s)\right] d s .
$$

Note that

$$
S(t):=\int_{\mathbb{R}^{N}} e^{-i t \cdot s}\left[s^{\gamma} D^{\gamma} k(s)\right] d s=\int e^{-i t \cdot s} h(s) \tilde{k}(s) d s
$$

where $h(\cdot):=\left\|(\cdot)^{\gamma} D^{\gamma} k(\cdot)\right\|_{\tau}$ is in the unit ball of $L_{1}\left(\mathbb{R}^{N} ; \mathbb{R}\right)$ and

$$
\tilde{k}(s):=[h(s)]^{-1} s^{\gamma} D^{\gamma} k(s) \in \tau_{0} .
$$

Therefore $S(t) \in \tau_{0}$. So there is a constant $C_{\alpha}$ so that $t^{\alpha} D^{\alpha} M(t) \in C_{\alpha} \tau_{0}$ for each $t \in \mathbb{R}^{N} \backslash\{0\}$. Now Theorem 3.2 gives the desired result.

\section{SMOOTH OPERATOR-VALUED FUNCTIONS}

The theorem in this section gives that the range of a sufficiently smooth function from $\mathbb{R}^{N}$ into $\mathcal{B}(X, Y)$ is R-bounded. Smoothness is measured in terms of the modulus of smoothness $s$ of a Besov space $B_{q, r}^{s}$. Also, the Fourier type of $Y$ is taken into account in order to reduce the required degree of smoothness. The theorem's corollaries measure smoothness in more classical ways (e.g. in terms of derivatives or a Lipschitz-like condition) rather than in terms of Besov spaces.

There are several equivalent well-known definitions of Besov spaces $B_{q, r}^{s}\left(\mathbb{R}^{N} ; Z\right)$; e.g. Besov spaces can be defined via a Paley-Littlewood decomposition (c.f., e.g., [18, Def. 2.4]), via real interpolation (c.f., e.g., [1]), or as in [31, Prop. 3.1]. 
THEOREM 5.1. Let $X$ be an arbitrary Banach space and $Y$ have Fourier type $p \in[1,2]$. Let $M \in B_{p, 1}^{N / p}\left(\mathbb{R}^{N} ; \mathcal{B}(X, Y)\right)$ have norm $A$. Then the set

$$
\tau:=\left\{M(t) \in \mathcal{B}(X, Y): t \in \mathbb{R}^{N}\right\}
$$

is R-bounded and $R_{1}(\tau) \leq C A$ for some constant $C$ that depends only on $\mathcal{F}_{p, N}(Y)$.

PROOF. First assume in addition that $M \in \mathcal{S}(\mathcal{B}(X, Y))$. Fix $x \in X$. Note that $[\widehat{M(\cdot) x}](t)=\widehat{M}(t) x$ since $M \in L_{1}$. Thus by [18, Cor. 3.2], for each $x \in X$,

$$
\int_{\mathbb{R}^{N}}\|\widehat{M}(t) x\|_{Y} d t \leq C_{1}\|M(\cdot) x\|_{B_{p, 1}^{N / p}\left(\mathbb{R}^{N} ; Y\right)} \leq C_{1} A\|x\|_{X}
$$

for some constant $C_{1}$ depending only on $\mathcal{F}_{p, N}(Y)$. By the Fourier inversion formula

$$
M(t)=(2 \pi)^{-N} \int_{\mathbb{R}^{N}} e^{i t \cdot s} \widehat{M}(s) d s \quad \text { for each } t \in \mathbb{R}^{N} .
$$

Applying Lemma 4.1 with $h_{t}(s)=(2 \pi)^{-N} e^{i t \cdot s}$ gives that

$$
R_{1}\left(\left\{M(t): t \in \mathbb{R}^{N}\right\}\right) \leq 2 C_{1} A .
$$

This proves the claim if $M \in \mathcal{S}(\mathcal{B}(X, Y))$.

For an arbitrary function $M \in B_{p, 1}^{N / p}\left(\mathbb{R}^{N} ; \mathcal{B}(X, Y)\right)$, choose a sequence $\left\{M_{k}\right\}_{k \in \mathbb{N}}$ from $\mathcal{S}(\mathcal{B}(X, Y))$ so that $\sum_{k=1}^{n} M_{k}$ converges to $M$ in $B_{p, 1}^{N / p}\left(\mathbb{R}^{N} ; \mathcal{B}(X, Y)\right)$ and

$$
\sum_{k \in \mathbb{N}}\left\|M_{k}\right\|_{B_{p, 1}^{N / p}} \leq 2\|M\|_{B_{p, 1}^{N / p}}
$$

Note that $\sum_{k=1}^{n} M_{k}$ converges to $M$ also in $L_{\infty}\left(\mathbb{R}^{N} ; \mathcal{B}(X, Y)\right)$ since the formal identity mapping from $B_{p, 1}^{N / p}\left(\mathbb{R}^{N} ; Z\right)$ into $L_{\infty}\left(\mathbb{R}^{N} ; Z\right)$ is continuous by the Sobolev embedding theorem. Thus

$$
\begin{aligned}
R_{1}\left(\left\{M(t): t \in \mathbb{R}^{N}\right\}\right) & \leq \sum_{k \in \mathbb{N}} R_{1}\left(\left\{M_{k}(t): t \in \mathbb{R}^{N}\right\}\right) \\
& \leq \sum_{k \in \mathbb{N}} 2 C_{1}\left\|M_{k}\right\|_{B_{p, 1}^{N / p}} \leq 4 C_{1}\|M\|_{B_{p, 1}^{N / p}}
\end{aligned}
$$

by [36, Lemma 2.4] and the above argument.

REMARK 5.2. In Theorem 5.1, the assumption that $M \in B_{p, 1}^{N / p}\left(\mathbb{R}^{N} ; \mathcal{B}(X, Y)\right)$ with norm $A$ can be replaced by a pointwise estimate; more precisely, it can be replaced by: $M$ is strongly integrable and

$$
\|M(\cdot) x\|_{B_{p, 1}^{N / p}\left(\mathbb{R}^{N} ; Y\right)} \leq A\|x\|_{X} \quad \text { for each } \quad x \in X .
$$

PROOF. For $x \in X$, define $M_{x}: \mathbb{R}^{N} \rightarrow Y$ by $M_{x}(\cdot):=M(\cdot) x$. Since $M$ is strongly integrable, the function $L: \mathbb{R}^{N} \rightarrow \mathcal{B}(X, Y)$, given by

$$
[L(t)](x):=\left(\mathcal{F} M_{x}\right)(t),
$$


is well-defined. By (5.1) and [18, Cor. 3.2], $\mathcal{F} M_{x} \in L_{1}(Y)$ with

$$
\left\|\widehat{M_{x}}\right\|_{L_{1}(Y)} \leq C A\|x\|_{X}
$$

for some constant $C$ that depends only on $\mathcal{F}_{p, N}(Y)$. Thus by the Fourier inversion formula

$$
M_{x}(t)=(2 \pi)^{-N} \int_{\mathbb{R}^{N}} e^{i t \cdot s} \widehat{M_{x}}(s) d s=\int_{\mathbb{R}^{N}} h_{t}(s)[L(s)](x) d s
$$

where $h_{t}(s):=(2 \pi)^{-N} e^{i t \cdot s}$. As in the proof of Theorem 5.1, Lemma 4.1 now gives the desired result.

The following corollary compares to [33, Thm. 4.1].

COROLLARY 5.3. Let $X$ be an arbitrary Banach space and $Y$ have Fourier type $p \in[1,2]$. Let $l=[N / p]+1$. Assume that $t \in \mathbb{R}^{N} \rightarrow M(t) \in \mathcal{B}(X, Y)$ satisfies

$$
\left(\int_{\mathbb{R}^{N}}\left\|D^{\alpha} M(t)\right\|_{\mathcal{B}(X, Y)}^{p} d t\right)^{1 / p} \leq A
$$

for each $\alpha \in \mathbb{N}_{0}$ with $|\alpha| \leq l$. Then $\left\{M(t) \in \mathcal{B}(X, Y): t \in \mathbb{R}^{N}\right\}$ is R-bounded.

PROOF. Since $N / p<l \in \mathbb{N}$, the formal identity map from a Sobolev space $W_{p}^{l}\left(\mathbb{R}^{N} ; Z\right)$ into $B_{p, 1}^{N / p}\left(\mathbb{R}^{N} ; Z\right)$ is continuous. Thus there are constants $K_{i}$ so that

$$
\begin{aligned}
\|M\|_{B_{p, 1}^{N / p}\left(\mathbb{R}^{N} ; \mathcal{B}(X, Y)\right)} & \leq K_{1}\|M\|_{W_{p}^{l}\left(\mathbb{R}^{N} ; \mathcal{B}(X, Y)\right)} \\
& :=K_{1} \sum_{0 \leq|\alpha| \leq l}\left\|D^{\alpha} M\right\|_{L_{p}\left(\mathbb{R}^{N} ; \mathcal{B}(X, Y)\right)} \leq K_{1} K_{2} A .
\end{aligned}
$$

Now apply Theorem 5.1.

Recall that for a function $\mathbb{R} \supset(a, b) \ni t \rightarrow M(t) \in \mathcal{B}(X, Y)$ with integrable derivative [36] (or more generally, for a function of bounded variation [33]) the set $\{M(t) \in \mathcal{B}(X, Y): t \in(a, b)\}$ is R-bounded. A short proof of this in the integrable derivative case follows easily from Lemma 4.1 since

$$
M(t)=M(a)+\int_{a}^{b} \chi_{[a, t]}(s) M^{\prime}(s) d s .
$$

The next corollary is a variant of these results. Its proof uses the following (equivalent) definition [31, Prop. 3.1] of Besov spaces $B_{p, 1}^{1 / p}(\mathbb{R} ; Z)$ for $p \in(1,2]$ :

$$
B_{p, 1}^{1 / p}(\mathbb{R} ; Z)=\left\{f \in L_{p}(\mathbb{R} ; Z): B_{p, 1}^{1 / p}(f)<\infty\right\},
$$

equipped with the norm $\|f\|_{B_{p, 1}^{1 / p}}^{\prime}=\|f\|_{L_{p}}+B_{p, 1}^{1 / p}(f)$, where

$$
B_{p, 1}^{1 / p}(f)=\int_{0}^{\infty} \sup _{|h| \leq s}\|f(\cdot+h)-f(\cdot)\|_{L_{p}(Z)} \frac{d s}{s^{1+\frac{1}{p}}} .
$$


COROLLARY 5.4. Let $X$ be an arbitrary Banach space and $Y$ have Fourier type $p \in(1,2]$. Fix $\alpha \in(1 / p, 1)$ and $\varepsilon \in(\alpha-1 / p, \alpha]$. If $M \in L_{p}(\mathbb{R} ; \mathcal{B}(X, Y))$ satisfies

$$
\|M(t+s)-M(t)\|_{\mathcal{B}(X, Y)} \leq A \frac{|s|^{\alpha}}{1+|s|^{\varepsilon}}(1+|t|)^{-\alpha} \quad \text { for each } \quad s, t \in \mathbb{R}
$$

for some constant $A$, then $\{M(t) \in \mathcal{B}(X, Y): t \in \mathbb{R}\}$ is R-bounded.

PROOF. Note that $\frac{|s|^{\alpha}}{1+|s|^{\varepsilon}}$ is increasing in $|s|$ since $\varepsilon \leq \alpha$. Thus for $s \geq 0$

$$
\begin{aligned}
\sup _{|h| \leq s}\left[\int_{R}\|M(t+s)-M(t)\|_{\mathcal{B}(X, Y)}^{p} d t\right]^{1 / p} & \leq A \frac{s^{\alpha}}{1+s^{\varepsilon}}\left[\int_{R}(1+|t|)^{-\alpha p} d t\right]^{1 / p} \\
& =A\left[\frac{2 p}{\alpha p-1}\right]^{1 / p} \frac{s^{\alpha}}{1+s^{\varepsilon}}
\end{aligned}
$$

since $1 / p<\alpha$. Note that

$$
\int_{0}^{\infty} \frac{s^{\alpha}}{1+s^{\varepsilon}} \frac{d s}{s^{1+\frac{1}{p}}} \leq \int_{0}^{17} s^{\alpha-1-\frac{1}{p}} d s+\int_{17}^{\infty} s^{\alpha-1-\frac{1}{p}-\varepsilon} d s .
$$

On the right-hand side of inequality (5.3), the first integral is finite since $1 / p<\alpha$ while the second integral is finite since $\alpha-1 / p<\varepsilon$. Thus $B_{p, 1}^{1 / p}(M)$ is finite and so $M \in B_{p, 1}^{1 / p}(\mathbb{R} ; \mathcal{B}(X, Y))$. Now apply Theorem 5.1.

Now for some applications to semigroup theory.

EXAMPLE 5.5. Let $\left(T_{t}\right)_{t>0}$ be a $c_{0}$-semigroup on a Banach space $X$ with generator $A$. Define

$\omega\left(T_{t}\right):=\inf \left\{w \in \mathbb{R}:\right.$ there exists a constant $C_{w}$ with $\left\|T_{t}\right\| \leq C_{w} e^{w t}$ for $\left.t>0\right\}$

$s_{R}(A):=\inf \{w \in \mathbb{R}:\{R(\lambda, A): \Re \lambda>w\}$ is R-bounded $\}$

$s_{N}(A):=\inf \{w \in \mathbb{R}:\{R(\lambda, A): \Re \lambda>w\}$ is norm bounded $\}$

$s(A):=\sup \{\Re \lambda: \lambda \in \sigma(A)\}$.

a) Then $s_{R}(A) \leq \omega\left(T_{t}\right)$; however, equality need not hold in general.

b) If furthermore $\left(T_{t}\right)_{t>0}$ is positive and $X$ is a $q$-concave Banach lattice for some $q \in[1, \infty)$, then $s(A) \stackrel{=}{=} s_{R}(A)$.

QUESTION 5.6. If $A$ generates a $c_{0}$-semigroup $\left(T_{t}\right)_{t \geq 0}$ on a Banach space $X$, then

$$
s(A) \leq s_{N}(A) \leq s_{R}(A) \leq \omega\left(T_{t}\right) .
$$

The first (resp. last) inequality in (5.4) need not be an equality in general, as illustrated by several examples already in the literature (resp. by Example 5.5 above). It is not know whether the middle inequality in (5.4) need not be an equality in general. 
Proof of Example 5.5 Towards part a), let $\Re \lambda>w_{1}>\omega\left(T_{t}\right)$. Then for each $x \in X$

$$
R(\lambda, A) x=\int_{0}^{\infty} e^{-\left(\lambda-w_{1}\right) t}\left[e^{-w_{1} t} T_{t} x\right] d t
$$

(cf., eg., [17, Thm. II.1.10]) and so it follows from Lemma 4.1 that

$$
\left\{R(\lambda, A): \Re \lambda>w_{1}\right\}
$$

is R-bounded. Hence $s_{R}(A) \leq \omega\left(T_{t}\right)$.

Now assume the setting of part b). Let $\Re \lambda \geq \lambda_{0}>s(A)$. Then

$$
|R(\lambda, A) x| \leq R\left(\lambda_{0}, A\right)|x|
$$

for each $x \in X$ (cf., eg., [4, C-III: Thm. 1.2, Cor. 1.3]); hence,

$$
\left\{R(\lambda, A): \Re \lambda>\lambda_{0}\right\}
$$

is R-bounded (just consider inequality (2.1) and [28, Prop. 1.d.9]) so $s(A)=s_{R}(A)$.

However, there are positive $c_{0}$-semigroups on $q$-concave Banach lattices ( $q$ finite) for which $\omega\left(T_{t}\right)>s(A)$ (cf., eg., [29, Ch. 4 Ex. 4.2]).

EXAMPLE 5.7. Let $-A$ be the generator of a $c_{0}$-semigroup $\left(T_{t}\right)_{t \geq 0}$ on a Banach space $X$ having Fourier type $p \in(1,2]$ and $\omega\left(T_{t}\right)<0$. Denote by $i_{\alpha}$ the embedding of $D\left(A^{\alpha}\right)$ with its graph norm into $X$. Then

$$
\left\{T_{t} i_{\alpha}: t \geq 0\right\} \subset \mathcal{B}\left(D\left(A^{\alpha}\right), X\right)
$$

is $\mathrm{R}$-bounded for $1 / p<\alpha<1$.

PROOF. Let $X_{\theta}$ be the real interpolation space $(X, D(A))_{\theta, 1}$. By [5, Thm. 6.7.3], the norm $\|x\|_{\theta}$ on $X_{\theta}$ is equivalent to

$$
\|x\|_{X}+\int_{0}^{\infty}\left(t^{-\theta} w(x, t)\right) \frac{d t}{t} \quad \text { where } \quad w(x, t):=\sup _{s \leq t}\left\|T_{s} x-x\right\| .
$$

Let $h_{x}(t):=T_{|t|}(x)$. Note that $h_{x} \in L_{1}(\mathbb{R} ; X)$ since $\omega\left(T_{t}\right)<0$. Furthermore, using the notation from (5.2),

$$
B_{p, 1}^{1 / p}\left(h_{x}\right) \leq\left(2 \int_{0}^{\infty}\left\|T_{u}\right\| d u\right)\left(\int_{0}^{\infty} t^{-1 / p} w(x, t) \frac{d t}{t}\right)
$$

and since $D\left(A^{\alpha}\right) \subset X_{1 / p}$ for $\alpha>1 / p$

$$
\left\|h_{x}\right\|_{B_{p, 1}^{1 / p}(X)} \leq C\|x\|_{X_{1 / p}} \leq C_{1}\|x\|_{D\left(A^{\alpha}\right)} .
$$

Now just apply Remark 5.2 with $M(t)=T_{|t|}$. 


\section{Bibliography}

1. Herbert Amann, Linear and quasilinear parabolic problems. Vol. I, Birkhäuser Boston Inc., Boston, MA, 1995, Abstract linear theory.

2. W. Arendt and S. Bu, The operator-valued Marcinkiewicz multiplier theorem and maximal regularity, (preprint).

3. __ Tools for maximal regularity, (preprint).

4. W. Arendt, A. Grabosch, G. Greiner, U. Groh, H. P. Lotz, U. Moustakas, R. Nagel, F. Neubrander, and U. Schlotterbeck, One-parameter semigroups of positive operators, Springer-Verlag, Berlin, 1986.

5. Jöran Bergh and Jörgen Löfström, Interpolation spaces. An introduction, Springer-Verlag, Berlin, 1976, Grundlehren der Mathematischen Wissenschaften, No. 223.

6. Earl Berkson and T. A. Gillespie, Spectral decompositions and harmonic analysis on UMD spaces, Studia Math. 112 (1994), no. 1, 13-49.

7. S. Blunck and P. C. Kunstmann, Weighted norm estimates and maximal regularity, (submitted).

8. Sönke Blunck, Maximal regularity of discrete and continuous time evolution equations, Studia Math. 146 (2001), no. 2, 157-176.

9. J. Bourgain, A Hausdorff-Young inequality for B-convex Banach spaces, Pacific J. Math. 101 (1982), no. 2, 255-262.

10. __ Some remarks on Banach spaces in which martingale difference sequences are unconditional, Ark. Mat. 21 (1983), no. 2, 163-168.

11. __ Vector-valued singular integrals and the $H^{1}-B M O$ duality, Probability theory and harmonic analysis (Cleveland, Ohio, 1983), Dekker, New York, 1986, pp. 1-19.

12. __ Vector-valued Hausdorff-Young inequalities and applications, Geometric aspects of functional analysis (1986/87), Springer, Berlin, 1988, pp. 239-249. 
13. P. Clément, B. de Pagter, F. A. Sukochev, and H. Witvliet, Schauder decomposition and multiplier theorems, Studia Math. 138 (2000), no. 2, 135-163.

14. Philippe Clément and Jan Prüss, An operator-valued transference principle and maximal regularity on vector-valued $L_{p}$-spaces, Evolution equations and their applications in physical and life sciences (Bad Herrenalb, 1998), Dekker, New York, 2001, pp. 67-87.

15. R. Denk, M. Hieber, and J. Prüß, R-boundedness, Fourier multipliers and problems of elliptic and parabolic type, (submitted).

16. Joe Diestel, Hans Jarchow, and Andrew Tonge, Absolutely summing operators, Cambridge University Press, Cambridge, 1995.

17. Klaus-Jochen Engel and Rainer Nagel, One-parameter semigroups for linear evolution equations, Springer-Verlag, New York, 2000, With contributions by S. Brendle, M. Campiti, T. Hahn, G. Metafune, G. Nickel, D. Pallara, C. Perazzoli, A. Rhandi, S. Romanelli and R. Schnaubelt.

18. Maria Girardi and Lutz Weis, Operator-valued Fourier multiplier theorems on Besov spaces, Mathematische Nachrichten, (to appear).

19. __ Operator-valued Fourier multiplier theorems on $L_{p}(X)$ and geometry of Banach spaces, (submitted).

20. Jerome A. Goldstein, Semigroups of linear operators and applications, The Clarendon Press Oxford University Press, New York, 1985.

21. R. Haller, H. Heck, and A. Noll, Mikhlin's theorem for operator-valued Fourier multipliers on $n$-dimensional domains, (preprint).

22. N. J. Kalton and G. Lancien, A solution to the problem of $L^{p}$-maximal regularity, Math. Z. 235 (2000), no. 3, 559-568.

23. N. J. Kalton and L. Weis, The $H^{\infty}$-calculus and sums of closed operators, Math. Ann. 321 (2001), no. 2, 319-345.

24. N. J. Kalton and Lutz Weis, Comparison and perturbation theorems for the $H^{\infty}$-calculus, (in preparation).

25. P. C. Kunstmann, Maximal $L_{p}$-regularity for second order elliptic operators with uniformly continuous coefficients on domains, (submitted). 
26. Peer Christian Kunstmann and Lutz Weis, Perturbation theorems for maximal $L_{p}$-regularity, Ann. Scuola Norm. Sup. Pisa Cl. Sci. (4) 30 (2001), no. 2, 415435.

27. S. Kwapień, Isomorphic characterizations of inner product spaces by orthogonal series with vector valued coefficients, Studia Math. 44 (1972), 583-595, Collection of articles honoring the completion by Antoni Zygmund of 50 years of scientific activity, VI.

28. Joram Lindenstrauss and Lior Tzafriri, Classical Banach spaces. II, SpringerVerlag, Berlin, 1979, Function spaces.

29. A. Pazy, Semigroups of linear operators and applications to partial differential equations, Springer-Verlag, New York, 1983.

30. Jaak Peetre, Sur la transformation de Fourier des fonctions à valeurs vectorielles, Rend. Sem. Mat. Univ. Padova 42 (1969), 15-26.

31. A. Pełczyński and M. Wojciechowski, Molecular decompositions and embedding theorems for vector-valued Sobolev spaces with gradient norm, Studia Math. 107 (1993), no. 1, 61-100.

32. Gilles Pisier, Some results on Banach spaces without local unconditional structure, Compositio Math. 37 (1978), no. 1, 3-19.

33. Ž. Štrkalj and Lutz Weis, On operator-valued Fourier multiplier theorems, (submitted).

34. Alberto Venni, Marcinkiewicz and Mihlin multiplier theorems, and $R$ boundedness, (preprint).

35. Lutz Weis, A new approach to maximal $L_{p}$-regularity, Evolution equations and their applications in physical and life sciences (Bad Herrenalb, 1998), Dekker, New York, 2001, pp. 195-214.

36. __ Operator-valued Fourier multiplier theorems and maximal $L_{p^{-}}$ regularity, Math. Ann. 319 (2001), no. 4, 735-758. 